\title{
¿Psicoanálisis y desarrollo? Aportes desde una perspectiva no
} lineal

\section{Psychoanalysis and Development? Contributions from a non-Linear Perspective}

Ariel Martínez $1 \bowtie \underline{\text { ORCID }}$

${ }^{1}$ Doctor en Psicología. Profesor en Universidad Nacional de la Plata. Investigador del Centro Interdisciplinario de Investigaciones en Género (CInIG), perteneciente al Instituto de Investigaciones en Humanidades y Ciencias Sociales (IdIHCS, UNLP/CONICET). Argentina.

Fecha correspondencia:

Recibido: noviembre 1 de 2019.

Aceptado: junio 15 de 2021.

Forma de citar:

Martínez, A. (2021). ¿Psicoanálisis y desarrollo? Aportes desde una perspectiva no lineal. Rev. CES

Psico, 14(3), 151-170.

https://dx.doi.org/10.21615/

cesp.5601

Open access

(C) Derecho de autor

Licencia creative commons

Ética de publicaciones

Revisión por pares

Gestión por Open Journal System

DOI: $10.21615 /$ cesp.5601

ISSNe: 2011-3080

Publica con nosotros

\section{Resumen}

El presente artículo problematiza la relación entre psicoanálisis freudiano y la perspectiva del desarrollo lineal, determinista y teleológica. A pesar de las impregnaciones evolucionistas del contexto histórico en que Freud gesta sus ideas, se encuentran conceptos que muestran una fuerte ruptura con la idea de un despliegue guiado por el ritmo de etapas prefijadas por edades. Así se postula la a-temporalidad de los procesos inconscientes y la Nachträglichkeit a la luz de las perspectivas no lineales emergentes desde las ciencias de la complejidad. Finalmente, se reflexiona sobre una psique que, en su carácter abierto y complejo, contempla lo aleatorio, lo impredecible y el azar en su devenir, y se transforma mediante trabajo elaborativo simbólico del yo. Así, sólo la historización, nunca lineal, contempla lo nuevo como posibilidad de (re)constituir la historia pasada.

Palabras claves: desarrollo, no linealidad, a-temporalidad, Nachträglichkeit, historización. 


\section{Abstract}

This article problematizes the relationship between Freudian psychoanalysis and the linear, deterministic, and teleological perspective on development. Despite the evolutionist impregnations of the historical context in which Freud conceives his ideas, there are concepts that show a sharp break with the idea of an unfolding guided by a rhythm of stages prefixed by ages. Thus, the a-temporality of the unconscious processes and the Nachträglichkeit is postulated considering the non-linear perspectives that emerge from the sciences of complexity. Finally, we reflect on a psyche that, in its open and complex character, contemplates the fortuitous, the unpredictable and chance in its becoming, and is transformed through the elaborative symbolic work on the self. Like this, only the historicization, which is never linear, contemplates what is new as a possibility to (re)construct the past history.

Keywords: development, non-linearity, a-temporality, Nachträglichkeit, historicization.

\section{Introducción}

Frecuentemente, suele delimitarse como rasgo general del desarrollo psicológico el cambio vinculado con la emergencia de novedades a partir de sistemas o estructuras previas, pero no contenidas en ellas (Lenzi, Borzi, \& Tau, 2010). Asimismo, forma parte de las perspectivas del desarrollo explicar los procesos que producen el surgimiento de esos cambios (Overton, 2003). Por otra parte, los cambios suponen un proceso temporal complejo y dinámico, vinculado con el carácter central de la irreversibilidad temporal que confronta la novedad emergente con lo previamente existente (Valsiner, 2006). En sus versiones más clásicas, vinculadas con derivas teleológicas del evolucionismo que se alejan del darwinismo (Burman, 1998), el proceso de desarrollo suele caracterizarse en términos de secuencia temporal de etapas, lo que implica cambio continuo y dirigido (Harris, 1957; Ghiselin, 1973). Entre sus principales componentes encontramos la especificación de un estado final de la organización, una descripción de las características finales del proceso de desarrollo. Un proceso de cambio, donde las características discretas están sujetas a transformaciones significativas o cambios cualitativos. Por lo tanto, típicamente, el desarrollo está en contraste con la repetición, o con los cambios cíclicos recurrentes. Así, encontramos referencias prospectivas del desarrollo que lo orienta, necesariamente, hacia el futuro. Los diversos momentos de desarrollo son precursores de etapas posteriores o últimas. El movimiento progresivo o acumulativo del desarrollo se vincula con un proceso secuencial que tiende hacia estructuras o funciones más complejas. Finalmente, se postula una integración jerárquica del desarrollo, que explica cómo elementos anteriores se incorporan en una organización mayormente integrada. Así, los elementos anteriores 
contribuyen, de alguna manera, al nuevo sistema emergente y, al mismo tiempo, se asimilarán de alguna manera en las nuevas estructuras.

Estos componentes del desarrollo, en su versión clásica, integran estructuras cuyo despliegue es lineal y determinista (Lewontin, \& Levins, 2007; Losos, \& Lenski, 2016). Actualmente, estas miradas clásicas han sido ampliamente contestadas por las miradas no lineales que conciben la forma en que los trayectos determinados se tensionan con aspectos azarosos y no predecibles (Doomen, 2011).

\section{Psicoanálisis y la exclusión de lo aleatorio}

Lo accidental, lo azaroso y los eventos aleatorios han incomodado al pensamiento occidental, incluido el psicoanálisis. Como forma de lidiar con el desorden se le ha adjudicado un orden oculto, se lo ha reducido a una manifestación secundaria del orden, o incluso ha sido desterrado por completo de los modelos explicativos. Como estrategia para dominar lo imprevisible, la racionalidad moderna segmenta dicotómicamente la realidad y desprecia unos de sus polos (Derrida, 1981). Así, ha proliferado una antítesis mutuamente excluyente entre, por un lado, el orden, la predictibilidad, el determinismo y la ciencia, y por el otro lado, el trastorno, la aleatoriedad y la naturaleza, vinculadas con la irracionalidad.

Sin embargo, en las primeras décadas del siglo XX, la mecánica cuántica plantea una aleatoriedad fundamental en el núcleo de la actividad subatómica. Pero la estrategia ante la aleatoriedad cuántica transcurre por, nuevamente, minimizarla. Las teorías no lineales recientes, incluidas las teorías del caos y la complejidad, han llegado más lejos. En estas propuestas, aleatoriedad y orden se entrelazan al punto de instalar el oxímoron del caos determinista: a la vez ordenado, en tanto es determinista, y desordenado en el sentido de incluir el azar. También señalan que, en los sistemas autoorganizados, la aleatoriedad juega un papel nodal en la producción de nuevas estructuras (Palis, 2002).

Es sabido que Freud y teóricos pertenecientes al campo del psicoanálisis presuponen un modelo de sistema que busca el equilibrio. En este modelo, la psique puede homologarse a un sistema sujeto a una tendencia a restablecer el equilibrio cuando éste se ve perturbado por un evento aleatorio (ya sea una fluctuación interna o una perturbación externa). Estos supuestos de búsqueda de equilibrio han llevado a ignorar o eliminar aspectos teóricos de la teoría psicoanalítica, vinculados con la aleatoriedad. Así, los aportes en torno a sistemas no lineales permiten una vía de acceso de lo aleatorio en el pensamiento psicoanalítico (Hornstein, 1993). 
La aleatoriedad se ha asociado con el azar, los accidentes, los eventos impredecibles, los procesos estocásticos, el desorden, la irregularidad y los acontecimientos fortuitos. La aleatoriedad se ha definido en contraste con los procesos deterministas y causales, es decir una irregularidad extrema sin ningún patrón discernible. Born definió por causalidad el modo en que una cosa depende de otra, y por determinismo aquella capacidad de anticipar eventos futuros en función de la relación causal (Pais, 1982; Freire \& Lehner, 2010). Por ejemplo, el lanzamiento de una moneda no es un evento determinista. No podemos anticipar cómo caerá. Esto no significa que el evento no sea causal. Hay fuerzas que hacen que la moneda caiga cara o cruz, pero debido a que no las conocemos, el lanzamiento de monedas es un evento aleatorio, una cuestión de azar. No importa si uno adopta una interpretación epistemológica u ontológica de la aleatoriedad: nuestra ignorancia colapsa lo ontológico en lo epistemológico, porque nuestra falta de conocimiento previo hace que el evento aleatorio sea, de todas formas, aleatorio, al menos desde nuestra perspectiva (García, 2007). Lo aleatorio también conserva el sentido de imprevisibilidad: la naturaleza inesperada del evento aleatorio.

Los postulados teóricos de la teoría psicoanalítica se han articulado a partir de la ciencia y la filosofía del siglo XIX. El matemático belga Quetelet buscó mostrar la forma en que la dinámica social se derivaba de los mismos principios de conservación que se encuentran en las ciencias físicas (Sánchez Carrión, 1999). Quetelet buscó reemplazar la contingencia con leyes generales. Denigró los eventos aleatorios como desviaciones dentro de un marco basado en el equilibrio (Sheynin, 1986). Las variaciones aleatorias se consideraron estadísticamente no significativas porque se vincularon con el principio de conservación de la energía mediante el cual los sistemas tienden a mantener constantes las cantidades de energía.

En el campo de la biología, Darwin afirmó que la evolución procede de variaciones que luego se seleccionan naturalmente debido a su función adaptativa. Al leer a Malthus, Darwin se sorprendió por la forma en que, durante la lucha por la existencia, se preservan las variaciones favorables y se destruyen las desfavorables. Para Darwin, entonces, podemos decir que estas variaciones fueron aleatorias en el sentido de que no fueron el resultado de un diseño emergente desde un intelecto divino, sino eventos no diseñados, cuya utilidad para el organismo era impredecible, solo conocidos después del hecho de haber sido seleccionados en función de la supervivencia (Egerton, 1973). Aquí, el azar podría producir un resultado positivo, no solo una aberración. El problema para Darwin era cómo erigir una teoría que fuera determinista pero que incluyera variaciones aleatorias en este sentido. Intensamente interesado en cuestiones filosóficas como el libre albedrío, Darwin recurrió al azar (Lightman, 2010). Las ideas de Darwin tuvieron gran impacto en Freud, sin embargo, no fue influenciado 
por la variación aleatoria como clave del desarrollo evolutivo. Hay una ausencia notable en la teoría de Freud de cualquier referencia a variaciones aleatorias. Esto se debe a la simpatía freudiana por Lamark, para quien la evolución no procede por variación y selección azarosa, sino directamente a través de la herencia de las características adquiridas (Ritvo, 1990). En una carta a Karl Abraham en 1917, Freud escribió:

El propósito es poner a Lamarck enteramente en nuestro terreno, y mostrar que su 'necesidad' que forma y transforma los órganos no es otra cosa que el poder de la representación inconsciente sobre el propio cuerpo (...). De esta manera quedaría explicada psicoanalíticamente la finalidad (...) quedarían establecidos dos grandes principios de la transformación que lleva al progreso: el principio mediante la adaptación del propio cuerpo, y el segundo, mediante la transformación del mundo externo (Freud/Abraham, 1979, p. 292-293).

Desde este punto de vista, la interpretación freudiana de los procesos lamarckianos de transformación no incluye la aleatoriedad. Freud no aborda explícitamente el problema de la aleatoriedad, pero puede deducirse a partir de sus conceptualizaciones sobre la psique y de sus puntos de vista sobre los factores accidentales, aparentemente aleatorios. La formulación temprana, junto a Breuer, del principio de constancia cuantitativo postula que el aparato psíquico se esfuerza por mantener la cantidad de excitación presente lo más baja posible o al menos mantenerlo en un nivel constante (Breuer \& Freud, 1895/1979). Este principio de constancia es equiparable con una tendencia psíquica hacia la estabilidad y el equilibrio. En este contexto parece convincente conjeturar que Breuer y Freud habrían asegurado que cualquier fluctuación o perturbación aleatoria que afecte a la psique -como excitaciones excesivas-debe ser descargada para mantener la constancia de energía psíquica en equilibrio.

Otra fuente de comprensión de Freud sobre lo aleatorio se puede encontrar en su estudio de los fallidos. Para Freud (1901/1979), las caídas, tropiezos y resbalones de la lengua, aparentemente aleatorios, no pueden interpretarse como deslices involuntarios y accidentales de la acción motora. Estos aparentes accidentes son formas de garantizar la realización de deseos inconscientes. Aquí, Freud, en efecto, había quitado la calidad aleatoria de tales eventos y los había interpretado como determinados inconscientemente. No existe un error, solo la revelación continua del pasado. Freud no da lugar a la contingencia, si los errores y accidentes son significativos, sólo lo son cuando dejan de ser errores y accidentes. Freud tomó una actitud habitual de la ciencia de su época: reemplazar el desorden por el orden, lo aleatorio por el determinismo. Como un científico arraigado, Freud no concibe la presencia de la aleatoriedad 
de la ciencia, incluido el psicoanálisis. De allí su preocupación por buscar leyes deterministas capaces de regular la presencia de lo accidental y lo aleatorio.

Las nuevas ciencias no lineales están reconceptualizando la relación del orden con el desorden. La investigación sobre el proceso de autoorganización demostró que los eventos aleatorios juegan un papel crucial, incluso necesario, en el desarrollo y la evolución de los sistemas y en la formación de nuevas estructuras. Los eventos aleatorios o el ruido amplificado demuestran la influencia de la perturbación. Los nuevos patrones de autoorganización son la manifestación de esta nueva correlación y coherencia en todo el sistema (García, 2007). Es debido a la aleatoriedad que las estructuras autoorganizadas son impredecibles. Sin embargo, sí es predecible que el sistema se autoorganice en las condiciones apropiadas, y esto implica cierto determinismo. Atlan (1998) ha señalado la interacción íntima y la interdependencia de los efectos constructivos y desorganizadores de la aleatoriedad durante el proceso de autoorganización.

En suma, la autoorganización requiere un sistema lo suficientemente redundante como para resistir el efecto desorganizador de los elementos aleatorios que impactan allí. Esto significa que los impactos aleatorios se incorporan en un nuevo orden. Un sistema incapaz de mantener su organización intacta, o que no es lo suficientemente redundante, no puede autoorganizarse. Esto implica que la organización y la desorganización no se contraponen y excluyen. En un sistema complejo, el cambio se produce a través de un proceso que incluye un componente importante de búsquedas o exploraciones aleatorias (Atlan, 1998).

Es cierto, Freud no hace lugar explícito a lo aleatorio, más bien explica los fenómenos psíquicos a partir de un orden determinado ${ }^{1}$. Sin embargo, las perspectivas no lineales que hacen lugar a lo aleatorio nos conducen hacia un espectro de conceptos donde el determinismo psíquico interjuega con aperturas hacia lo novedoso, algo muy próximo al modo en que lo aleatorio establece relaciones de alianza con los aspectos permanentes de los sistemas y, así, engendran transformaciones que escapan al puro determinismo (Kornblihtt, 1994).

El pensamiento freudiano gesta, colateralmente, herramientas conceptuales que nos permiten incluir lo aleatorio en sus ideas. Estos conceptos resisten, sintomáticamente, a que el psicoanálisis pueda ser reducido a los modelos del desarrollo clásicos cuando intentamos dar

\footnotetext{
${ }^{1}$ Esta afirmación sobre el carácter determinista del pensamiento freudiano no hace justicia a la totalidad de referencias bibliográficas existentes al respecto. Existen otros puntos de vista que permiten complejizar la perspectiva presentada (Lahitte, Azcona, \& Oria, 2013; Ortiz Vanegas, 2018).
} 
cuentas de las transformaciones del sujeto a lo largo del tiempo (Bernardi, 1994). Cuando nos interrogamos sobre cómo poner en diálogo estos aspectos o dimensiones del desarrollo con la teoría psicoanalítica, y si consideramos la mirada panorámica de la propuesta freudiana, la complejidad metapsicológica del psicoanálisis complica las cosas cuando queremos asimilar su propuesta con cualquier modelo lineal.

Como señala Jane Flax (1995), la vastedad de la teoría psicoanalítica constituye un pensamiento fragmentario donde la ambivalencia, positivamente valorada desde el prisma posmodernista, enfrenta la potencia del pensamiento freudiano con las narrativas del desarrollo impregnadas, en mayor o menor medida, por la matriz del evolucionismo (Anandalakshmy \& Grinder, 1970). En tanto pensamiento articulado a finales del siglo XIX, el psicoanálisis conserva marcas de época y ofrece elementos que nos permiten proyectar secuencias del desarrollo (Ghiselin, 1973). Aun así, como hemos señalado, el psicoanálisis se resiste a quedar epistemológicamente absorbido en los componentes del desarrollo ya mencionados. Nos interesa utilizar la perspectiva no lineal referida para alentar un traslado que se dirige desde los intentos por restringir el psicoanálisis a un relato linealmente progresivo y ordenado mediante cambios transformacionales secuenciales, hacia una delimitación de los conceptos psicoanalíticos que nos permiten pensar las transformaciones del sujeto a lo largo del tiempo por fuera de cualquier modelo lineal, determinista y teleológico. Es posible problematizar la noción de desarrollo desde un rastreo de ideas freudianas en las cuales encontramos ecos de lo aleatorio (Hornstein, 1994). Encontramos posible deslindar algunas conceptualizaciones freudianas que interrumpen los supuestos implicados en las perspectivas del desarrollo, estas son: la deconstrucción retroactiva de la indestructibilidad de lo previo -nos permitirá pensar una modalidad de cambio que hace lugar a lo aleatorio- y la a-temporalidad de lo inconsciente -nos permitirá pensar el orden y la permanencia, un requerimiento necesario para la supervivencia de cualquier sistema.

\section{Deconstrucción retroactiva de la indestructibilidad de lo previo}

Desde la propuesta teórica freudiana, la noción de desarrollo (particularmente el desarrollo sexual) tropieza frente a una noción retroactiva del tiempo. Para Freud, ciertos eventos adquieren sentido en un desfase temporal. Emplea el término Nachträglich para referir a la asignación de un significado nuevo a rastros de memoria. Estos rastros de memoria están sujetos, de vez en cuando, a nuevos arreglos, nueva transcripción (Freud, 1897/1979), en función de nuevas circunstancias. El uso ha ido amplificándose actualmente, y se utiliza para referir a la transferencia retroactiva de significado sobre eventos pasados a través de la lente del presente. Laplanche, por ejemplo, refiere a la Nachträglichkeit en términos de dos escenas 
unidas por cadenas asociativas, pero separadas entre sí por un lapso temporal que las inscribe en dos esferas de significado diferentes $(2001,2012)$.

Freud no adoptó la comprensión convencional del tiempo como una sucesión cronológica en la que el pasado, el presente y el futuro se suceden y se desplazan mutuamente. Sí le preocupó cómo, psíquica e históricamente, el pasado puede entenderse paradójicamente como un momento que aún no ha llegado y aún no ha finalizado, una oportunidad disponible para la actualización o alteración. En una carta a Fliess Freud señala: "tú sabes que trabajo con el supuesto de que nuestro mecanismo psíquico se ha generado por estratificación sucesiva, pues de tiempo en tiempo el material preexistente de huellas mnémicas experimenta un reordenamiento según nuevos nexos, una retrascripción" (1897/1979, p. 274). Es a esto a lo que Freud denomina efecto nachträglich en el orden del tiempo. Al respecto, Laplanche y Pontalis aportan la siguiente definición: "Palabra utilizada frecuentemente por Freud en relación con su concepción de la temporalidad y la causalidad psíquica: experiencias, impresiones y huellas mnémicas son modificadas ulteriormente en función de nuevas experiencias (...). Entonces pueden adquirir (...) un nuevo sentido" (1983, p. 280).

Esta compleja estructura temporal se compone de (al menos) dos escenas separadas en el tiempo, algo así como una temporalidad bifásica de la memoria que Freud vincula con la producción del trauma. La acción diferida de la Nachträglichkeit se aleja de cualquier forma simple de determinismo lineal. La posibilidad de que lo actual altere el sentido de lo previo, tornándolo traumático, requiere de un período de latencia -antes de que la alteración del significado pueda tener lugar- $y$, fundamentalmente, la capacidad inconsciente del aplazamiento temporal del significado. El inconsciente asedia el flujo temporal irreversible del desarrollo mediante impactos traumáticos que marcan discontinuidades y alejan la experiencia subjetiva de cualquier linealidad posible.

La característica distintiva de la Nachträglichkeit reside en la apertura de los sentidos inconscientes. Las vivencias actuales proyectan sentidos retroactivamente y así alteran el sentido bajo el cual el recuerdo fue registrado psíquicamente. Esta incongruencia temporal, que nos desfasa en un demasiado antes o en un demasiado después, es condición para una nueva comprensión (Freud, 1897; Freud \& Breuer, 1895), para la resignificación. La diferencia entre un momento temporal demasiado temprano y un momento temporal demasiado tarde abre una brecha de diferencia donde anida esta extraordinaria temporalidad de un presente capaz de re-articular el pasado. En el primer momento estructural de la Nachträglichkeit, llegar demasiado pronto a la experiencia escénica significa habitar un mundo de significantes sin las 
claves que nos permiten acceder a sus significados. El segundo momento estructural implica comprender el significado después de la primera experiencia escénica. Los sentidos a partir de los cuales registramos las experiencias siempre son superficiales, pues es posible suplementarlos con capas de significación que anexan retroactivamente una densidad que jamás totalizan o clausuran sus posibilidades. La segunda escena nos abre el significado de lo que ha sucedido antes, algo así como una iluminación retroactiva de la propia historia.

La Nachträglichkeit dirige la flecha del tiempo, simultáneamente, en dos direcciones. El tiempo se presenta no como una secuencia lineal, sino como una dinámica que produce nuevos significados. Laplanche (2012) nos recuerda que, si solemos pensar que el futuro es una apertura a todas las posibilidades y que el pasado esta clausurado como aquello irremediablemente perdido, Freud sugiere que el pasado está abierto a rearticulaciones que nos permite volver a trazar nuevas posibilidades desde donde proyectarnos a un futuro que, a priori, se encuentra clausurado bajo los sentidos cristalizados en nuestras escenas psíquicas tempranas.

Si bien esta temporalidad distorsionada de la Nachträglichkeit fue acuñada por Freud para conceptualizar la temporalidad que involucra, de forma distintiva, la producción del trauma, llama la atención que James Strachey lo ha traducido como acción diferida, lo que tiende a minimizar la potencia y alcance conceptual, como si Nachträglichkeit se limitara a un simple diferimiento temporal o a un período de latencia. Sin embargo, más que el impacto diferido de los eventos pasados en las circunstancias presentes, la Nachträglichkeit se refiere al impacto del presente en el pasado, lo que sugiere que la temporalidad psíquica no solo se despliega linealmente hacia adelante sino también hacia atrás, retroactivamente. Es cierto que este concepto implica una reacción diferida o retardada que, más tarde, reaviva o despierta nuevas experiencias, estímulos o traumas, pero debe quedar claro que Freud rechazó definitivamente la causalidad lineal del tiempo del desarrollo. Después de todo, como señala Andree Green (2000) este carácter retroactivo agita la memoria inconsciente y los afectos asociados a ella, dándole nueva vida y significado al registro psíquico de experiencias previas.

Jean Laplanche ha hecho de Nachträglichkeit o l'après-coup el centro de su explicación de la subjetividad. Señala la forma en que ciertos acontecimientos de la infancia pueden inscribirse de manera relativamente difusa en alguna parte de la psique sin adquirir todo su sentido e, incluso, sin tener prácticamente sentido alguno para el sujeto. Estos recuerdos quedan allí en estado de huellas mnémicas informes, para posteriormente cristalizar, adquirir sentido $y$ esclarecer retrospectivamente el pasado. 
Como hemos señalado, las concepciones tradicionales de desarrollo se centran en el modo en que se transforman e incluyen elementos previos en la nueva organización, más compleja y global. Esta complejidad creciente es factible gracias a la posibilidad de incorporar lo previo, el pasado. La mirada psicoanalítica nos enfrenta con la compulsión a la repetición y la pulsión de muerte (Freud, 1920/1979), dinámicas que recrean insistentemente el pasado, un retorno a objetos y metas previas. En sentido estricto, este pasado no necesita ser alcanzado o revivido, pues siempre persistió, en su forma original, en el revés del presente. A diferencia de la Nachträglichkeit, el tiempo cronológico, sobre el cual cabalga el desarrollo, no transforma ni afecta el pasado, así quedan inalterados muchos elementos antiguos. El pasado está exento de tiempo, es "indestructible" (Freud, 1900/1979, p. 608), "virtualmente inmortal" (Freud, $1933 / 1979$, p. 69), por lo tanto, no puede ser descartado o borrado, tampoco integrado o subsumido en otras estructuras más complejas. La vida psíquica conserva sus elementos antiguos, elecciones de objetos, metas pulsionales y marcas, inscripciones, huellas mnémicas de las experiencias más tempranas subsisten inalteradas.

Desde un punto de mira psicoanalítico, el pasado determina el presente y moldea el futuro en gran medida -la Nachträglichkeit contrarresta la fuerza del determinismo-, esto se debe, precisamente, a que todas las experiencias tempranas decisivas en la constitución psíquica se conservan en su forma original:

de las formaciones anímicas infantiles nada sucumbe en el adulto a pesar de todo el desarrollo posterior. Todos los deseos, mociones pulsionales, modos de reaccionar y actitudes del niño son pesquisables todavía presentes en el hombre maduro (...). No están destruidos, sino situados bajo unas capas que se les han superpuesto (...). Así, se convierte en un carácter del pasado anímico no ser devorado por sus retoños, como lo es el histórico; persiste junto a lo que devino desde él, sea de una manera sólo virtual o en una simultaneidad real (Freud, 1913/1979, p. 186).

Bajo la forma espacial, topológica, en que Freud figura el inconsciente para nuestra comprensión, las marcas y huellas configuran un registro acumulativo de experiencias. Los residuos psíquicamente eficaces de este pasado mental se manifiestan constantemente en nuestro despliegue subjetivo. Sin embargo, el pasado no parece configurar una fuente de determinismo inexorable. La posibilidad de investir psíquicamente un tiempo futuro debe concebir la posibilidad de un desajuste, de una no coincidencia (Aulagnier, 2004). El futuro no consta de un reencuentro con el pasado -mal que le pese a nuestra economía narcisista. Freud nos habla de un enfrentamiento con un "eco de esta fase del desarrollo" (Freud, 1905, p. 208). 
Se trata de ecos, lo que parece darnos un espectro de posibilidades y de improvisación, claro que en un campo de restricciones constitutivas (Butler, 2008).

\section{A-temporalidad de lo inconsciente}

En el contexto de la teoría psicoanalítica, las nociones de repetición, retorno e indestructibilidad de las primeras inscripciones de las experiencias tempranas apartan la realidad psíquica de los efectos de la dimensión del tiempo prospectivo -uno de los principales vectores de desarrollo. La dinámica retroactiva de la vida psíquica requiere de alguna territorialidad topológica donde el movimiento temporal cronológico se suspende. Es imposible negar avances en el desarrollo, pero las fuerzas anteriores, preservadas y no erosionadas por el paso del tiempo irrumpen continuamente y llegan a dominar la escena psíquica. Por lo tanto, la a-temporalidad caracteriza la concepción psicoanalítica del modo en que las transformaciones se incrustan en una concepción temporal que desborda el carácter secuencial del desarrollo.

La a-temporalidad es señalada claramente al final de la Interpretación de los sueños: "En el inconsciente, a nada puede ponerse fin, nada es pasado ni está olvidado" (Freud, 1900/1979, p. 569). Los procesos inconscientes "son atemporales, es decir, no están ordenados con arreglo al tiempo, no se modifican por el trascurso de este ni, en general, tienen relación alguna con él" (Freud, 1915/1979, p. 184). Desde aquí surge una nueva forma de olvido activo no vinculado con procesos temporales sino inconscientes:

suele creerse que es el tiempo el que vuelve inciertos y deslíe los recuerdos (...) respecto del olvido no se pueda hablar de una función directa del tiempo (...) En el caso de las huellas mnémicas reprimidas, se puede comprobar que no han experimentado alteraciones durante los más largos lapsos. Lo inconsciente es totalmente atemporal (Freud, 1901/1979, p. 266).

La suspensión del tiempo o el vacío de tiempo que envuelve las manifestaciones del inconsciente las despoja de cualquier significado diacrónico. Para el psicoanálisis, el tiempo del inconsciente es una ficción psicológica que no podemos dejar de proyectar cuando intentamos abordar su lógica específica.

No es posible obviar, en este punto, la famosa Nota sobre la 'pizarra mágica' o el block maravilloso que Freud escribió en 1925. Allí explica la compleja forma en que se depositan los 
recuerdos o huellas mnémicas en una superficie que, como la pizarra mágica², los conserva incólumes. La capacidad de recepción de la superficie de escritura resulta inagotable. En los dispositivos de registro -en los que solemos apoyar nuestra memoria- que usan tinta sobre un papel, o tiza sobre una pizarra, la "capacidad ilimitada de recepción y conservación de huellas duraderas parecen excluirse (...) o bien es preciso renovar la superficie receptora, o bien hay que aniquilar los signos registrados" (Freud, 1925/1979, p. 243-244). Aún más interesante, Freud nos dice: "nuestro aparato anímico opera lo que ellos no pueden: es ilimitadamente receptivo para percepciones siempre nuevas, y además les procura huellas mnémicas duraderas -aunque no inalterables" (Freud, 1925/1979, p. 244, la cursiva es mía). Freud encuentra que la pizarra mágica reúne el modo en que funciona el aparato psíquico, cuenta con "una superficie perceptiva siempre dispuesta [percepción consciente que no genera huellas duraderas] y huellas duraderas de los caracteres recibidos [sistema mnémico inconsciente donde las huellas tienen lugar permanente]" (Freud, 1925/1979, p. 244). Nuestro registro mnémico, al igual que la lámina encerada, conserva la nitidez de las marcas, "huella duradera de lo escrito, legible con una iluminación adecuada" (Freud, 1925/1979, p. 245).

Los modelos no lineales y complejos arrojan luz sobre una organización psíquica que aloja la aleatoriedad y lo impredecible. También muestra estrecha conexión con la modalidad temporal retroactiva de los procesos psíquicos. Desde aquí, el psicoanálisis ofrece un marco teórico epistemológico desde el cual abordar las transformaciones del sujeto a lo largo del tiempo que no se ajusten a la perspectiva de la psicología del desarrollo.

\section{Hacia el despliegue no lineal del yo}

El psicoanálisis norteamericano ofrece elementos teóricos capaces de concebir la psique a la luz de la dinámica no lineal y de lo aleatorio sin que esto implique el derrumbe de su funcionamiento continuo. Desde aquí, la psique se entiende como un sistema autoorganizado que requiere aleatoriedad para la aparición de nuevas estructuras y patrones, un elemento necesario para la comprensión de los cambios psíquicos. La mirada norteamericana ha puesto énfasis en el yo y su función sintética (Wallerstein, 1998). El yo, diferenciado del Ello, alcanza

\footnotetext{
2 Freud describe la pizarra mágica como "una tablilla de cera o resina de color oscuro, (...) hay sobre ella una hoja delgada, trasparente, fija (...) [estos] dos estratos (...) pueden separarse entre sí, salvo en ambos márgenes trasversales. El de arriba es una lámina trasparente de celuloide, y el de abajo, un delgado papel encerado, también trasparente. Cuando el aparato no se usa, la superficie inferior del papel encerado adhiere levemente a la superficie superior de la tablilla de cera. Para usar esta pizarra mágica, se trazan los signos sobre la lámina de celuloide de la hoja que recubre a la tablilla de cera (...) se produce por mediación de la hoja que sirve de cubierta. El punzón, en los lugares que toca, hace que la superficie inferior del papel encerado oprima la tablilla de cera, y estos surcos se vuelven visibles, como una escritura de tono oscuro, sobre la superficie clara y lisa del celuloide. Si se quiere destruir el registro, basta con tomar el margen inferior libre de la hoja de cubierta, y separarla de la tablilla de cera mediante un ligero movimiento. De ese modo cesa el íntimo contacto entre papel encerado y tablilla de cera en los lugares rasgados (...) y no vuelve a establecerse cuando ambas se tocan de nuevo. Ahora la pizarra mágica ha quedado libre de toda escritura y preparada para recibir nuevos registros" (Freud, 1925/1979, p. 244-245).
} 
un alto grado de organización, el cual le resulta necesario para mantener su tendencia a unificarse a pesar del flujo temporal (Rappaport, Enrich, \& Wilson, 1982). Finalmente, el yo reúne la capacidad de defenderse del efecto desorganizador de las mociones pulsionales, pues liga excitaciones y reprime objetos inadmisibles a la conciencia.

Heinz Hartmann se ocupó directamente de explicar las diversas funciones sintéticas del yo. El autor enfatiza la forma en que el ego organiza y controla la motilidad y la percepción, también sirve como una barrera protectora contra el exceso de excitaciones externas y de estímulos internos (Leichtman, 1990). En suma, el yo es una verdadera organización, pues mantiene el equilibrio. Hartmann señaló explícitamente su similitud con el concepto de homeostasis (Sabelli et. al., 1997). A esta función sintética, teorizada por el psicoanálisis norteamericano del ego, subyace la preocupación por el equilibrio. En sentido estricto, la organización del yo no sintetiza, más bien excluye de la psique lo que es incompatible con su equilibrio. En un modelo excesivamente centrado en el equilibrio resulta difícil comprender la posibilidad de sus cambios. Si el yo sintetizador restablece continuamente el equilibrio cada vez que la psique es perturbada, ésta es arrastrada continuamente a su estado actual y, de este modo, abraza continuamente la permanencia. La función defensiva del yo lo mantiene en una zona libre de conflictos, zona entendida en términos de homeostasis (Marcus, 1999). Este modelo de funcionamiento es dominado por una tendencia a buscar el equilibrio luego de fluctuaciones o perturbaciones aleatorias internas o externas.

Los sistemas no lineales nos enfrentan con procesos de autoorganización que conciben el efecto desorganizador y reorganizador de la aleatoriedad, y la posibilidad de una síntesis superior. Esto nos conduce hacia una conceptualización del yo que persigue el cambio, la novedad y la transformación. La síntesis emergente, a partir de su enfrentamiento con lo aleatorio, toma el orden pasado e incorpora el evento aleatorio en un nuevo orden emergente, sintético y estable. La autoorganización dentro del marco de la teoría de sistemas dinámicos no lineales concibe tanto la estabilidad como la inestabilidad necesaria para el cambio. La inestabilidad caracterizaría a la psique cuando emergen bifurcaciones. Un yo que excluye continuamente la aleatoriedad no necesita de de una zona libre de conflictos para sintetizar operaciones, ya que son precisamente los conflictos los que evocan el potencial autoorganizador de un sistema.

El yo, entonces, en un marco de autoorganización, integra la aleatoriedad y el orden. Adam Phillips propone un yo contingente como alternativa al yo psicoanalítico clásico cuyos aspectos inconscientes muestran un determinismo fuerte. Para Phillips, entonces, los cambios refieren 
con una creciente capacidad para apropiarse, utilizar y capitalizar coincidencias aleatorias. La autoorganización de la psique es factible debido a la contingencia del yo, su posibilidad de transformarse en un proceso continuo en el que la novedad tiene lugar a medida que el sistema se ve afectado e incorpora eventos aleatorios (Meredith-Owen, 1995). El yo autoorganizado contiene potenciales de transformación inherentes a la no linealidad interactiva con sus elementos y procesos.

Capaz de ser afectada por la aleatoriedad, la psique autoorganizada -proceso posibilitado por la dinámica retroactiva de la Nachträglichkeit- escapará de un determinismo absoluto -que emana del núcleo permanente garantizado por la a-temporalidad de lo inconsciente. La psique autoorganizadora es creativamente transformada y tiende hacia el cambio. El yo puede formularse como el lugar de síntesis mediante el cual lo aleatorio puede conducir a reorganizaciones y al cambio. No es que lo aleatorio llegue a dominar de forma absoluta al sistema, sino que habrá períodos de estabilidad y períodos de inestabilidad cuando lo aleatorio llegue a tener un efecto crítico. La psicoanalista francesa Piera Aulagnier (2004) refiere a la actividad del yo en términos de historiador. El yo se autoconstruye a sí mismo, de forma continua, sobre aquel lugar nuevo emergente de los múltiples encuentros entre temporalidades heterogéneas (Najmanovich, 1994). Cada uno de estos encuentros configura un origen, produce singularidad y (re)creación del yo.

La paradójica dinámica inconsciente nos enfrenta con la paradójica referencia a procesos atemporales. Sylvie Le Poulichet (1996) señala al respecto que el juego libre de la energía característica de los procesos primarios, donde todo deviene y nada cesa, no puede devenir pasado. La dinámica incesante de la pulsión, presencia moviente, configura una masa de acontecimientos que no cesa. Del lado de los procesos inconscientes nada cesa y nada desaparece. Del lado del yo los acontecimientos pueden resultar pasados, ordenados, representados mediante el examen de realidad. La actividad de construir una historia, en parte, es posible porque el yo -aprendiz de historiador- se empeña en representar el tiempo, asigna a los acontecimientos un orden temporal, pero los procesos inconscientes -maestro brujogeneran acontecimientos en la insistencia de lo que no cesa (Aulagnier, 2003). La atemporalidad de lo inconsciente es crucial para el proceso que el yo despliega en tanto historiador, allí se produce la insistencia permanente de los acontecimientos que no cesan, que no devienen pasados, pero que, sin embargo, no permanecen inmóviles e idénticos. Es gracias a que los acontecimientos pueden transformarse y desplegarse en los procesos inconscientes, bajo el sello de indestructibilidad, que el yo puede anudar un tiempo sin historia y una historia sin tiempo. Mediante la puesta en historia, el yo se rescata a sí mismo de un devenir anónimo. 
Queda claro el modo en que el aporte freudiano extrae la dimensión del pasado de cualquier consideración cronológica. Parafraseando a Lacan (1979), la psique lleva el pasado, siempre y en todo lugar, pegado a la suela de sus zapatos.

Cuando Freud afirma que durante el trabajo de análisis "lo deseable es una imagen confiable, e íntegra en todas sus piezas esenciales, de los años olvidados de la vida del paciente" (Freud, $1937 / 1979$, p. 260), nos enfrenta con una operación que lejos de intentar completar una versión del pasado ofrece espacio para una narración sobre el pasado que resulta la construcción de una historia para el yo. El yo, mediante su trabajo de historiador, se re-sitúa respecto a un pasado que, en ese mismo acto, construye (Aulagnier, 1991a). Este proceso es condición de posibilidad para proyectarse en la apertura psíquica de un tiempo futuro. Construir una historia implica un trabajo psíquico activo y singular por parte del yo a partir del cual se producen nuevos sentidos (Lewkowicz, 1997). A partir de la elaboración simbólica, el yo anuda a representaciones aquellos acontecimientos que no cesa de producirse. En relación con el trabajo que el yo despliega como historiador, Piera Aulagnier señala: "es una necesidad de su funcionamiento situarse y anclar en una historia que sustituye un tiempo vivido-perdido por la versión que el sujeto se procura merced a su reconstrucción de las causas que lo hicieron ser, que dan razón de su presente y hacen pensable un eventual futuro" (Aulagnier, 2003, p 15).

La configuración y proyección en un tiempo futuro se apoya en una reconstrucción siempre parcial, fragmentaria, selectiva, continua y cambiante. Pero el yo construye una versión de sus orígenes, de su propio pasado enunciado y anticipado por los otros, en suma: debe anclarse en referencias identificatorias permanentes y estables que aseguren la continuidad y la transformación a lo largo del tiempo (Aulagnier, 1991a). La permanencia de la historia sin tiempo de lo inconsciente signada por la pura repetición asegura un punto estable para que el sujeto no se diluya en la vertiente dinámica de un puro flujo temporal sin historia. En aquel cruce el yo se articula en su proceso de construir una historia, condición ineludible para su despliegue, y así construye una distancia irreductible y estructurante entre un tiempo pasado y un tiempo actual, o futuro anticipado. La actividad del yo despliega trabajos de subjetivación de experiencias que suplementan la trama actual de representaciones construidas acerca del pasado -en el revés de cuyas representaciones late la insistencia repetitiva de las marcas de nuestra pura historia, paradójicamente, a-temporal.

Ante la a-temporalidad del inconsciente donde no hay pasado, Piera Aulagnier (2004) nos deja en claro el requerimiento ineludible de construir un pasado para que la existencia misma del yo, sean posibles. Pero este origen que el yo necesita representarse no debe diluirse sin más en 
perspectivas del desarrollo. André Green señala críticamente "la tendencia a reducir el modelo temporal complejo de Freud al mero punto de vista genético (...), el concepto (...) de Nachtraglichkeit (...) hace estallar los modelos temporales clásicos" (Green, 2000 p.21). Aún más, en relación con el origen, Sylvie Lepoulichet nos dice:

el origen es lo que uno no cesa de deducir cuando se actualiza un tiempo de recomposición, y ello hasta el punto de producirse en un tiempo reversivo. El origen es este momento fuera del tiempo, inaprensible, que excede a todo comienzo. Lo que toma figura de origen es la interpretación del comienzo, autorizando la constitución de una historia que no es ni el pasado ni lo vivido. Y si algo 'nuevo' se inscribe una y otra vez en una vida, es por cuanto el origen, simultáneamente, se recompuso (1996, p. 84).

Es claro que, en la lógica de los procesos psíquicos, se trastorna la relación causal clásica a tal punto que "los efectos continúan componiendo sus propias causas" (Le Poulichet, 1996, p. 89). Piera Aulagnier (1991a, 2003, 2004) toma la dinámica temporal de la Nachträglichkeit y señala el modo en que el yo deviene mediante recomposiciones de sus orígenes y sus proyectos. Nos muestra cómo el trabajo del yo intenta, como condición de su existencia y devenir, hacer frente a la tarea titánica de lidiar con una de las manifestaciones de los procesos psíquicos inconscientes: "el no-lugar del origen" (Le Poulichet, 1996, p. 92). El trabajo retroactivo de construir(se) un pasado (Aulagnier, 1991a) que despliega el yo es el intento de construir y mantener la certeza de un origen pasado para poder devenir en un tiempo futuro, una dinámica alejada de la linealidad y teleología de los modelos temporales clásicos.

\section{Comentarios finales}

El psicoanálisis postula la a-temporalidad de los procesos inconscientes, esto asegura la indestructibilidad de sus inscripciones y huellas mnémicas, pero también la temporalidad retroactiva, denominada Nachträglichkeit, nos garantiza la posibilidad de transformar lo previo. Hemos visto cómo esta dinámica temporal rompe con la linealidad y el determinismo de las miradas clásicas del desarrollo. Si admitimos el aporte del psicoanálisis freudiano, los modelos lineales no alcanzan para explicar exhaustivamente las transformaciones del sujeto a lo largo del tiempo, pues dejan fuera aquellos aspectos de la subjetividad en las que el tiempo funciona por fuera de la cronología, aquella tópica donde el pasado opera como una permanencia que, lejos de pensarse como pasado cronológico debe considerarse como anterioridad lógica.

La dinámica del inconsciente nos señala un aspecto de la subjetividad que no puede integrarse en el flujo secuencial del tiempo que distingue entre el pasado y el presente. La Nachträglichkeit 
es posible en tanto la permanencia de lo inconsciente opera como principio de permanencia (Aulagnier, 1991b), como núcleo estable que garantiza el cambio. La Nachträglichkeit rompe con el determinismo y, así fractura la linealidad. Más importante aún, esta dinámica temporal requiere de la acción de lo aleatorio, puesto que la escena actual a partir de la cual se altera lo previo requiere de la dimensión azarosa que los sistemas lineales excluyen. La tarea del yo como historiador sólo cobra sentido cuando la estructura psíquica conserva un núcleo permanente a partir del cual es posible pensar el cambio y la transformación. La temporalidad psíquica admite el impacto de lo imprevisible y lo aleatorio. Las transformaciones del sujeto a lo largo del tiempo operan entre el doble principio de permanencia y cambio (Aulagnier, 1991b), donde el determinismo opera de forma constante y el azar de forma puntual (Hornstein, 1994). Sólo un sistema capaz de transformar lo ya constituido abraza la posibilidad de pensar transformaciones radicalmente nuevas, no determinadas linealmente. Aunque fuertemente evolucionista, a finales del siglo XIX, el genio freudiano logró suspender la pregnancia de Lamark para ofrecernos procesos que permiten explicar cambios que van más allá de los términos clásicos, lineales y deterministas que pueblan la psicología del desarrollo.

\section{Referencias}

Anandalakshmy, S., \& Grinder, R. (1970). Conceptual Emphasis in the History of Developmental Psychology: Evolutionary Theory, Teleology, and the Nature-Nurture Issue. Child Development, 41(4), 1113-1123. https://www.jstor.org/stable/1127338

Atlan, H. (1998). Intentional Self-Organization. Emergence and Reduction: Towards a Physical Theory of Intentionality. Thesis Eleven, 52(1), 5-34. https://doi.org/10.1177/0725513698052000003

Aulagnier, P. (1991a). Construir(se) un pasado. Revista de Psicoanálisis APdeBA, 13(3), 441-497. Aulagnier, P. (1991b). Los dos principios del funcionamiento identificatorio, permanencia y cambio. En L. Hornstein y otros (Comp.). Cuerpo, Historia, Interpretación. Buenos Aires: Paidós.

Aulagnier, P. (2003). El aprendiz de historiador y el maestro brujo. Del discurso identificante al discurso delirante. Buenos Aires: Amorrortu.

Aulagnier, P. (2004). La violencia de la interpretación. Del pictograma al enunciado. Buenos Aires: Amorrortu.

Bernardi, R. (1994). Sobre el determinismo psíquico. En S. Bleichmar (Comp.). Temporalidad, determinación, azar. Lo reversible y lo irreversible. Buenos Aires: Paidós.

Burman, E. (1998). La deconstrucción de la Psicología Evolutiva. Madrid: Visor.

Butler, J. (2008). Cuerpos que importan. Sobre los límites materiales y discursivos del 'sexo'. Buenos Aires: Paidós.

Derrida, J. (1981). Dissemination. University of Chicago Press.

Doomen, J. (2011). Cornering 'Free Will'. The Journal of Mind and Behavior, 32(3), 165-179. 
Egerton, F. (1973). Changing Concepts of the Balance of Nature. The Quarterly Review of Biology, 48(2), 322-350. https://www.jstor.org/stable/2820544

Flax, J. (1995). Psicoanálisis y Feminismo. Pensamientos Fragmentarios. Madrid: Cátedra.

Freire, O., \& Lehner, C. (2010). 'Dialectical materialism and modern physics', an unpublished text by max born. Notes and Records of the Royal Society of London, 64(2), 155-162. https://www.jstor.org/stable/20753890

Freud, S. (1897 [1979]). Fragmentos de la correspondencia con Fliess. En J. L. Etcheverry (Traduc.). Obras completas: Sigmund Freud (Vol. 1). Buenos Aires: Amorrortu.

Freud, S. (1900 [1979]). La interpretación de los sueños (segunda parte). En J. L. Etcheverry (Traduc.). Obras completas: Sigmund Freud (Vol. 5). Buenos Aires: Amorrortu.

Freud, S. (1901 [1979]). Psicopatología de la vida cotidiana (Sobre el olvido, los deslices en el habla, el trastrocar las cosas confundido, la superstición y el error). En J. L. Etcheverry (Traduc.). Obras completas: Sigmund Freud (Vol. 6). Buenos Aires: Amorrortu.

Freud, S. (1905 [1979]). Tres ensayos de teoría sexual. En J. L. Etcheverry (Traduc.). Obras completas: Sigmund Freud (Vol. 7). Buenos Aires: Amorrortu. (Trabajo original publicado en 1905).

Freud, S. (1913 [1979]). El interés por el psicoanálisis. En J. L. Etcheverry (Traduc.). Obras completas: Sigmund Freud (Vol. 13). Buenos Aires: Amorrortu.

Freud, S. (1915 [1979]). Lo inconciente. En J. L. Etcheverry (Traduc.). Obras completas: Sigmund Freud (Vol. 14). Buenos Aires: Amorrortu.

Freud, S. (1920 [1979]). Más allá del principio de placer. En J. L. Etcheverry (Traduc.). Obras completas: Sigmund Freud (Vol. 18). Buenos Aires: Amorrortu.

Freud, S. (1925 [1979]). Nota sobre la «pizarra mágica». En J. L. Etcheverry (Traduc.). Obras completas: Sigmund Freud (Vol. 19). Buenos Aires: Amorrortu.

Freud, S. (1933 [1979]). Nuevas conferencias de introducción al psicoanálisis. En J. L. Etcheverry (Traduc.). Obras completas: Sigmund Freud (Vol. 22). Buenos Aires: Amorrortu.

Freud, S. (1937 [1979]). Construcciones en el análisis. En J. L. Etcheverry (Traduc.). Obras completas: Sigmund Freud (Vol. 23). Buenos Aires: Amorrortu.

Freud, S., \& Breuer, J. (1895 [1979]). Estudios sobre la histeria. En Obras Completas, Tomo II. Buenos Aires: Amorrortu.

Freud, S./Abraham, K. (1979). Carta del 11 de noviembre de 1917. En Correspondencia. Barcelona: Gedisa.

García, R. (2007). Sistemas complejos. Conceptos, método y fundamentación epistemológica de la investigación interdisciplinaria. Barcelona: Gedisa.

Ghiselin, M. (1973). Darwin and Evolutionary Psychology. Science, New Series, 179(4077), 964968. https://science.sciencemag.org/content/179/4077/964

Green, A. (2000). El tiempo fragmentado. Buenos Aires: Amorrortu.

Harris, D. B. (1957). The Concept of Development. Minneapolis: University of Minnesota Press. 
Honig, R. G. (2005). Psychoanalysis and Developmental Theory. Journal of the American Psychoanalytic Association, 53(4), 1383-1388.

https://doi.org/10.1177/00030651050530040801

Hornstein, L. (1993). Historia, azar, determinismo. Hacia un nuevo paradigma. En Práctica psicoanalítica e historia. Buenos Aires: Paidós.

Hornstein, L. (1994). Determinismo, temporalidad y devenir. En S. Bleichmar (Comp.).

Temporalidad, determinación, azar. Lo reversible y lo irreversible. Buenos Aires: Paidós.

Kornblihtt, A. (1994). Biología molecular: consecuencias evolutivas del azar y de los procesos

reversibles e irreversibles. En S. Bleichmar (Comp.). Temporalidad, determinación, azar. Lo reversible y lo irreversible. Buenos Aires: Paidós.

Lacan, J. (1979). Escritos 1. México: Siglo XXI.

Lahitte, H. B., Azcona, M., \& Oria, V. O. (2013). La noción de causalidad en Sigmund Freud.

Límite. Revista Interdisciplinaria de Filosofía y Psicología, 8(27), 59-74. https://www.redalyc.org/pdf/836/83628140004.pdf

Laplanche, J. (2001). Entre seducción e inspiración: el hombre. Buenos Aires: Amorrortu.

Laplanche, J. (2012). El après-coup. Problemáticas VI. Buenos Aires: Amorrortu.

Laplanche, J., \& Pontalis, J. B. (1983). Diccionario de Psicoanálisis. Barcelona: Labor.

Le Poulichet, S. (1996). La obra del tiempo en psicoanálisis. Buenos Aires: Amorrortu.

Leichtman, M. (1990). Developmental Psychology and Psychoanalysis: I. the Context for a

Revolution in Psychoanalysis. Journal of the American Psychoanalytic Association, 38(4), 915950. https://doi.org/10.1177/000306519003800403

Lenzi, A., Borzi, S., \& Tau, R. (2010). El concepto de desarrollo en psicología: entre la evolución y la emergencia. Fundamentos en Humanidades, XI(II), 137-161. http://sedici.unlp.edu.ar/handle/10915/29087

Lewkowicz, I. (1997). Historización en la adolescencia. Cuadernos APdeBA, 1, 109-126.

Lewontin, R., \& Levins, R. (2007). Biology Under the Influence: Dialectical Essays on the Coevolution of Nature and Society. New York University Press.

Lightman, B. (2010). Darwin and the Popularization of Evolution. Notes and Records of the Royal Society of London, 64(1), 5-24. https://doi.org/10.1098/rsnr.2009.0007

Losos, J., \& Lenski, R. (2016). How Evolution Shapes Our Lives: Essays on Biology and Society. Princeton University Press.

Marcus, E. R. (1999). Modern Ego Psychology. Journal of the American Psychoanalytic Association, 47(3), 843-871. https://doi.org/10.1177/00030651990470031501

Meredith-Owen, W. (1995). Phillips, Adam. 'Contingency for Beginners'. Winnicott Studies. (1995). Journal of Analytical Psychology, 40(3), 492-493. https://www.pepweb.org/document.php?id=joap.040.0492a

Najmanovich, D. (1994). De 'EL TIEMPO' a las temporalidades. En S. Bleichmar (Comp.). Temporalidad, determinación, azar. Lo reversible y lo irreversible. Buenos Aires: Paidós. 
Ortiz Vanegas, J. (2018). Azar y libertad. Universidad Nacional de La Plata). http://sedici.unlp.edu.ar/bitstream/handle/10915/86955/Documento completo.pdfPDFA.pdf?sequence $=1$

Overton, W. F. (2003). Development across the life span. En R. M. Lerner, M. A. Easterbrooks, \& J. Mistry (Eds.). Comprehensive handbook of psychology: Developmental Psychology. New York: John Wiley \& Sons.

Pais, A. (1982). Max Born's Statistical Interpretation of Quantum Mechanics. Science, 218(4578), 1193-1198. https://science.sciencemag.org/content/218/4578/1193

Palis, J. (2002). Chaotic and complex systems. Current Science, 82(4), 403-406. https://www.jstor.org/stable/24106651

Rappaport, H., Enrich, K., \& Wilson, A. (1982). Ego Identity and Temporality: Psychoanalytic and Existential Perspectives. Journal of Humanistic Psychology, 22(4), 53-70. https://doi.org/10.1177/002216788202200405

Ritvo, L. (1990). Darwin's Influence on Freud: A Tale of Two Sciences. Yale University Press.

Sabelli, H., Carlson-Sabelli, L., Patel, M., \& Sugerman, A. (1997). Dynamics and Psychodynamics: Process Foundations of Psychology. The Journal of Mind and Behavior, 18(2/3), 305-334. https://www.jstor.org/stable/43853826

Sánchez Carrión, J. (1999). Presentación Quetelet y la Sociología. Reis: Revista Española de Investigaciones Sociológicas, 87 , 291-303. http://www.reis.cis.es/REIS/PDF/REIS 087 12.pdf

Sheynin, O. (1986). Quetelet as a Statistician. Archive for History of Exact Sciences, 36(4), 281325. https://link.springer.com/article/10.1007/BF00357247

Valsiner, J. (2006). Development epistemology and implications for methodology. En W. Damon y R. Lerner (Eds.). Handbook of child psychology. New York: Wiley.

Wallerstein, R. S. (1998). Erikson's Concept of Ego Identity Reconsidered. Journal of the American

Psychoanalytic Association, 46(1), 229-248. https://doi.org/10.1177/000306519804600112 